\title{
The clinical diagnosis of osteoporosis: a position statement from the National Bone Health Alliance Working Group
}

\author{
E. S. Siris • R. Adler • J. Bilezikian • M. Bolognese • B. Dawson-Hughes • M. J. Favus • \\ S. T. Harris • S. M. Jan de Beur • S. Khosla • N. E. Lane • R. Lindsay • A. D. Nana • \\ E. S. Orwoll $\cdot$ K. Saag $\cdot$ S. Silverman $\cdot$ N. B. Watts
}

Received: 23 January 2014 / Accepted: 11 February 2014 / Published online: 28 February 2014

(C) The Author(s) 2014. This article is published with open access at Springerlink.com

\begin{abstract}
Summary Osteoporosis causes an elevated fracture risk. We propose the continued use of T-scores as one means for diagnosis but recommend that, alternatively, hip fracture; osteopenia-associated vertebral, proximal humerus, pelvis, or some wrist fractures; or FRAX scores with $\geq 3 \%$ (hip) or $20 \%$ (major) 10-year fracture risk also confer an osteoporosis diagnosis.

Introduction Osteoporosis is a common disorder of reduced bone strength that predisposes to an increased risk for fractures in older individuals. In the USA, the standard criterion
\end{abstract}

\author{
E. S. Siris $(\bowtie) \cdot J$. Bilezikian \\ Division of Endocrinology, Department of Medicine, Columbia \\ University Medical Center, New York, NY, USA \\ e-mail: es27@cumc.columbia.edu \\ R. Adler \\ School of Medicine, Virginia Commonwealth University, Richmond, \\ VA, USA \\ M. Bolognese \\ Bethesda Health Research, Bethesda, MD, USA \\ B. Dawson-Hughes \\ Jean Mayer USDA Human Nutrition Research Center on Aging, \\ Bone Metabolism Laboratory, Tufts University, Boston, MA, USA \\ M. J. Favus \\ Department of Medicine, University of Chicago, Chicago, IL, USA \\ S. T. Harris \\ Department of Endocrinology and Metabolism, University of \\ California, San Francisco, CA, USA \\ S. M. Jan de Beur \\ Division of Endocrinology, Johns Hopkins University, Baltimore, \\ MD, USA \\ S. Khosla \\ Mayo Clinic, Rochester, MN, USA
}

for the diagnosis of osteoporosis in postmenopausal women and older men is a T-score of $\leq-2.5$ at the lumbar spine, femur neck, or total hip by bone mineral density testing.

Methods Under the direction of the National Bone Health Alliance, 17 clinicians and clinical scientists were appointed to a working group charged to determine the appropriate expansion of the criteria by which osteoporosis can be diagnosed.

Results The group recommends that postmenopausal women and men aged 50 years should be diagnosed with osteoporosis if they have a demonstrable elevated risk for future fractures.

N. E. Lane

School of Medicine, University of California, Davis, CA, USA

R. Lindsay

Helen Hayes Hospital, West Haverstraw, NY, USA

A. D. Nana
University of North Texas Health Science Center, Fort Worth, TX,
USA

E. S. Orwoll

Oregon Health \& Science University, Portland, OR, USA

K. Saag

Division of Clinical Immunology and Rheumatology, University of Alabama, Birmingham, AL, USA

\author{
S. Silverman \\ Bone Health Center, Cedars-Sinai Medical Center, Los Angeles, CA, \\ USA \\ N. B. Watts \\ Osteoporosis and Bone Health Services, Mercy Health Physicians, \\ Cincinnati, OH, USA
}


This includes having a T-score of less than or equal to -2.5 at the spine or hip as one method for diagnosis but also permits a diagnosis for individuals in this population who have experienced a hip fracture with or without bone mineral density (BMD) testing and for those who have osteopenia by BMD who sustain a vertebral, proximal humeral, pelvic, or, in some cases, distal forearm fracture. Finally, the term osteoporosis should be used to diagnose individuals with an elevated fracture risk based on the World Health Organization Fracture Risk Algorithm, FRAX.

Conclusions As new ICD-10 codes become available, it is our hope that this new understanding of what osteoporosis represents will allow for an appropriate diagnosis when older individuals are recognized as being at an elevated risk for fracture.

Keywords Clinical diagnosis · Criteria · Osteoporosis · Position statement

\section{Introduction}

Osteoporosis has been characterized as a skeletal disorder of reduced bone strength that leads to an increased risk for fracture, typically in the setting of low trauma such as a fall from standing height. In the USA today, the standard criterion for defining and diagnosing osteoporosis and applying the ICD-9 code 733.0 is the finding of a T-score of $\leq-2.5$ at the lumbar spine, femur neck, or total hip by bone mineral density (BMD) testing [1]. As T-scores decrease, the relative risk for fracture increases. This principle makes the T-score an effective means of identifying those individuals at increased fracture risk and offers a cut point that allows for a diagnosis of osteoporosis. However, it is clear that there are other ways to identify individuals at high fracture risk, including the occurrence of one or more of several types of low-trauma fractures or through the use of fracture risk algorithms such as FRAX. It has been suggested that either of these ways of predicting an increased fracture risk should also enable the use of the diagnostic term osteoporosis [2]. It is the purpose of this paper to make the case that we should formalize this concept and encourage clinicians to use the term osteoporosis when they identify an older patient with an elevated fracture risk determined by any one of these criteria.

Osteoporosis is a public health concern that is associated with over two million fractures per year in the USA [3]. This disease continues to be underdiagnosed, and its management with a variety of treatments, including adequacy of calcium and vitamin $\mathrm{D}$, exercise to improve balance and prevent falls, and pharmacologic therapy as indicated to lower fracture risk, remains suboptimal [3]. The diagnosis of osteoporosis based on a T-score of $\leq-2.5$ is and should remain one important way to identify an individual with an increased risk for fracture.
Bone density testing is recommended based on age and risk factor status in both men and women by the Surgeon General's Report on bone health and osteoporosis as well as other guidelines [3-6], but only a small proportion of older men and women have a BMD test [7]. Many who do receive the test may still not be recognized as having an elevated fracture risk because their scores reflect "osteopenia," which in some instances does indicate a high risk based on elevated age or prior fracture history or other validated risk factors. Prior fracture affords the highest risk for future fracture [4], yet an older patient with a hip fracture may not be diagnosed as having osteoporosis unless the patient has a BMD test with a $\mathrm{T}$-score of $\leq-2.5$, and the majority of hip fracture patients have T-scores that are better than -2.5 [8]. An incident vertebral fracture strongly predicts an increased risk of another vertebral fracture as soon as within the next year [9]. Several other fracture types also increase the risk of future fracture [10], and about half of patients with a hip fracture have already had a previous fracture [11], yet the term osteoporosis is not formally applied unless the BMD T-score is $\leq-2.5$. Most fractures occur in people with low bone mass, not "T-score" osteoporosis, because a greater number of people have osteopenia than osteoporosis as defined by BMD [12, 13]. The failure to detect clinical osteoporosis when it is present likely contributes to the current lack of awareness of the consequences of this disease by both clinicians and patients, impacts the reimbursement strategies of payers, influences policy makers in the public health sector by underestimating the number of those at elevated fracture risk, and affects the design of clinical trials of new agents to reduce fracture risk by both pharmaceutical companies and the FDA.

In a position paper published in 2012 [2], a recommendation was made to formally expand the criteria for allowing a diagnosis of osteoporosis to include the presence of certain low-trauma fractures or the determination of an elevated fracture risk using FRAX, without a T-score of -2.5 or lower. A proposal was made to assemble a task force of representatives from the academic and clinical societies that represent the bone field and osteoporosis in particular in the USA, to debate the issue and to reach consensus on the clinical characteristics that would allow and support a clinical diagnosis of osteoporosis in all older individuals who have an elevated risk for fracture.

\section{Working group composition and charge}

Under the direction of the National Bone Health Alliance (NBHA), a group of 17 clinicians and clinical scientists were appointed to a Clinical Diagnosis of Osteoporosis Working Group. Three of the members are the three US-based authors of the 2012 position paper [2]; six individuals were appointed 
by the National Osteoporosis Foundation (NOF), and six were appointed by the American Society for Bone and Mineral Research (ASBMR). One additional member is the representative to the NBHA of the American Academy of Orthopedic Surgeons, and the final member is the Centers for Disease Control Liaison to the NBHA. The committee carried out its work by meeting initially at the 2012 ASBMR annual meeting and, thereafter, through several teleconferences. There was no financial support by any entity for the committee's work, and full financial disclosure of the members is provided in this document.

The committee set a series of principles in place to be clear about its purpose. First, the population for whom the clinical osteoporosis diagnostic criteria are intended include (a) postmenopausal women and (b) men over the age of 50 in the USA. This population is the same as that included in the NOF Clinician's Guide [3]. Second, the purpose of expanding the criteria for making a diagnosis is limited to that; no specific treatment recommendations are being suggested as a part of this effort. While the committee agreed that some form of management would likely apply in all persons diagnosed as having osteoporosis by these new criteria (including the use of anti-osteoporosis prescription medication in many but not all cases), the sole purpose of this committee's deliberations is to expand the criteria by which osteoporosis can be diagnosed. Third, in this report, the terms osteopenia and low bone mass are being used interchangeably and refer to a bone mineral density T-score at the spine, femur neck, or total hip that is $<$ -1.0 and $>-2.5$. Finally, the fractures we are considering as being diagnostic for osteoporosis are low-trauma or lowenergy fractures, such as those occurring in our population from, as an example, a fall from standing height. Fractures resulting from major trauma, e.g., automobile accidents or falls off a roof, are not the fractures we are including in our definition, though in this population, it is still recommended that a bone density test be performed in such individuals to determine if low bone mass or osteoporosis as defined by $\mathrm{T}$ score is present.

The committee recognized that making a diagnosis of a condition is easiest when there are absolute criteria, such as a blood test result above or below a certain cut point or a biopsy of a lesion that has a clear pathological finding. However, often in medicine, we make a diagnosis based on clinical characteristics that reflect a syndrome that requires a diagnosis in order to devise a treatment plan. A solid evidence base coupled with physician judgment in some instances is necessary, and a thoughtful clinician needs to consider whether or not certain clinical characteristics reflect an underlying abnormality and to proceed with management accordingly. Clearly, it is our intent to allow the diagnosis of osteoporosis to be made when there appears to be evidence for believing that an elevated risk for fracture is present but, equally, to afford the opportunity to not use the term if it is not appropriate. Thus, in some instances where our recommendations for making the diagnosis of osteoporosis might apply in some cases but not in others, we have given examples that may be helpful.

\section{Committee recommendations}

Fracture types that allow a diagnosis of osteoporosis

There was a consensus that for the population under consideration, an individual who experiences a low-trauma hip fracture can be diagnosed with osteoporosis, with or without a BMD test. There was also a consensus that a low-trauma clinical vertebral fracture, proximal humerus fracture, or pelvis fracture is diagnostic of osteoporosis in a person with osteopenia. The incidental finding of a vertebral fracture on a radiograph (a morphometric vertebral fracture) may also be considered as diagnostic of osteoporosis if the clinician has a reason to believe that it is likely to have been the result of low bone mass and reduced bone strength. For example, if an older woman has one or more vertebral deformities discovered on a spine radiograph or through vertebral fracture assessment on a DXA test and cannot recall whether or not she had an episode of severe back pain but has BMD-based osteopenia, the osteoporosis diagnosis can be applied. However, if a 51-year-old man has a chest radiograph that shows a vertebral compression deformity and the patient recalls a bad sports injury at the age of 20 with weeks of severe back pain that gradually resolved and a current BMD shows normal or minimally low T-scores, the diagnosis of osteoporosis should not be made.

Finally, in some instances, a low-trauma distal forearm fracture in a person with osteopenia at the lumbar spine or hip by BMD testing may be considered as diagnostic of osteoporosis. A minority of the members of the working group felt that in the population being considered, any wrist fracture in the setting of osteopenia should be diagnosed as osteoporosis. Their argument is that anyone over 50 years who breaks a wrist regardless of the level of trauma and whose assessment reveals low bone mass has osteoporosis, because weaker bones are more likely to break than stronger bones, and wrist fractures predict future fractures [10, 14]. However, a majority of the working group felt that the circumstances of the wrist fracture must be taken into account, and patient age and the level of low bone mass be considered before labeling the patient as having osteoporosis. For example, if a 50-yearold woman crashes while roller blading sustaining a wrist fracture and has normal or minimally low BMD when that indicated test is performed (T-scores no lower than -1.3 , as an example), the diagnosis of osteoporosis is probably not appropriate. However, if a 64-year-old individual with a T-score of -2.2 slips on a curb, falls, and breaks her wrist, the 
diagnosis might apply, particularly if other risk factors are present. In the case of wrist fracture, the diagnosis may be made in some cases and not in others. Clinician judgment, in the context of the clinician understanding on the definition of osteoporosis, would need to be applied, as often is the case in the real world of clinical practice.

While many other fracture types increase the risk of future fractures [10], the committee did not feel that all fracture types, beyond those listed here, would necessarily constitute a basis for an osteoporosis diagnosis. Therefore, at this time, we suggest limiting the fracture types for an osteoporosis diagnosis to the hip, spine, proximal humerus, pelvis, and, in some cases, wrist, requiring a BMD test showing osteopenia at the spine or hip in all cases except hip fracture. There was also considerable discussion about the level of trauma that led to the fracture, noting that the classical definition of a "fragility" or "low-trauma" or "low-energy" fracture implies, for example, a fall from standing height. Regardless of the level of trauma, however, current recommendations call for a measurement of BMD in older individuals who sustain a fracture in order to rule in or out the possibility that low bone mass was a contributing factor [3].

The role of FRAX in making the diagnosis of osteoporosis

FRAX is a World Health Organization-sponsored, countryspecific fracture risk assessment tool that combines BMD at the femoral neck (or total hip) with a group of well-validated and weighted clinical risk factors for fracture that are largely independent of BMD [15]. It is based upon epidemiological data from 60,000 women and men studied prospectively to correlate risk factors for fracture with fracture outcomes and then validated in independent cohorts including more than 230,000 patients. It is useful as a way of predicting the risk of hip fracture and major osteoporotic fractures, i.e., clinical spine, hip, proximal humerus, and distal forearm fractures, in previously untreated men and women aged 40-90 years. Its use in the USA allows the assessment of fracture risk in both genders and four ethnic groups and is recommended primarily for individuals with a BMD finding of osteopenia [3].

There was consensus that for the population under consideration if the 10 -year probability of hip fracture is $\geq 3 \%$ or the 10 -year probability of major osteoporotic fracture is $\geq 20 \%$, a diagnosis of osteoporosis can be made. These two cut points reflect treatment intervention thresholds in the USA as described in the NOF Clinician's Guide, based in the case of hip fracture on cost-effectiveness criteria for hip fracture management [16], and were viewed as a logical basis for making a diagnosis of osteoporosis.

\section{Further considerations}

The committee believes that expanding the diagnostic criteria for osteoporosis will assist in properly identifying a greater number of people who are, in fact, at an elevated risk for fracture, thus increasing awareness and encouraging strategies to lower risk, which may or may not include prescription therapy in all cases but would be recommended by current guidelines for most [3]. The efficacy of many of the currently available therapies to lower fracture risk is based upon clinical trials in which entry criteria typically required BMD T-scores of $\leq-2.5$ at the spine or hip, not fracture history or FRAX scores. In several studies, vertebral fractures were a part of the entry criteria [17-19], with average T-scores reflecting osteopenia in many cases, and in other cases, FRAX data were applied post hoc to determine the efficacy based upon baseline FRAX scores [20, 21]. Individual decisions regarding treatment interventions with medications intended to lower fracture risk will be necessary, and clinicians are advised to consider whether there is evidence for a treatment effect for each of the various therapies in the absence of a T-score diagnosis of osteoporosis [2].

The committee also cautions that patients not be overdiagnosed based upon these recommendations. We acknowledge that fractures that occur with a high level of trauma may or may not have been influenced by the level of bone strength, and an evaluation, including BMD testing in most cases, is needed after the fracture to help determine the role that reduced bone strength may have played. Care should be taken to avoid making a diagnosis of osteoporosis if the fractures truly resulted from the severity of the trauma in the presence of fairly normal bones. Conversely, a fracture in an older individual after a fall from standing height is too often blamed on the fall without due consideration of the strength of the bones, and it is our hope that these new criteria will compel a more thoughtful assessment of overall fracture risk, so that underdiagnosis will also be reduced.

\section{Conclusion}

We recommend that postmenopausal women and also men over the age of 50 years should be diagnosed as having osteoporosis if it is demonstrated that the individual is at an elevated risk for future fractures. We support the continued use of BMD testing and the finding of a T-score of $\leq-2.5$ at the spine or hip as one way to make the diagnosis. We further support the use of the term osteoporosis for individuals in this population who have experienced a low-trauma hip fracture and for those who have osteopenia by BMD who sustain a low-trauma vertebral, proximal humerus, pelvis, or, in some cases, distal forearm fracture. Hip, vertebral, distal radius, and pelvis fractures constitute about two thirds of osteoporosis- 
associated fractures [22], and proximal humerus fractures are among the four "major osteoporotic fractures" together with hip, spine, and distal forearm fractures in FRAX [15]. Finally, for individuals who have an elevated fracture risk based on FRAX, the term osteoporosis can be used for diagnosis.

We hope to formalize these principles through future interactions with third-party payers as well as with our colleagues in primary care and orthopedics. As the new ICD-10 codes become available in 2014-2015, it is our hope that this new understanding on what osteoporosis represents will allow for its appropriate diagnosis when older individuals are recognized as being at an elevated risk for fracture.

Acknowledgments The working group acknowledges the expert and valuable advice from $\mathrm{Dr}$. Anne Looker of the $\mathrm{CDC}$ who serves as a liaison to the NBHA and also thanks Dr. Mia Lowden and Dr. Taylor Wallace of the NBHA who provided staff support for this effort. Finally, we thank Mr. David Lee, Executive Director of NBHA, for his commitment and untiring support for the working group endeavor.

Conflicts of interest ESS is a consultant for Amgen, Eli Lilly, Merck, Novartis, and Pfizer. RA has no conflicts to disclose. JB is a consultant for Amgen, Lilly, Merck, NPS, Bristol Meyers Squibb, and Johnson \& Johnson and has research grants from Amgen, NPS, and NIH. MB has no conflicts to disclose. BDH is on the scientific advisory board of Eli Lilly and Agnovos, is a consultant for Agnovos and Servier, and has research grants from Agnovos and Servier. MJF has no conflicts to disclose. STH is a consultant for Amgen, Eli Lilly, Gilead Sciences, and Merck. SMJ has no conflicts to disclose. SK is on the scientific advisory board of Amgen and Bone Therapeutics. NEL has no conflicts to disclose. RL has no conflicts to disclose. ADN has no conflicts to disclose. ESO is on the scientific advisory board of Merck, Eli Lilly, and Wright Medical Tech and has research grants from Amgen, Merck, and Eli Lilly. KS has no conflicts to disclose. SS has no conflicts to disclose. NBW has no conflicts to disclose.

Open Access This article is distributed under the terms of the Creative Commons Attribution Noncommercial License which permits any noncommercial use, distribution, and reproduction in any medium, provided the original author(s) and the source are credited.

\section{References}

1. NIH Consensus Development Panel on Osteoporosis Prevention, Diagnosis, and Therapy (2001) NIH consensus development panel on osteoporosis prevention, diagnosis and treatment. Osteoporosis prevention, diagnosis, and therapy. JAMA 285(6):785-795

2. Siris ES, Boonen S, Mitchell PJ, Bilezikian J, Silverman S (2012) What's in a name? What constitutes the clinical diagnosis of osteoporosis? Osteoporos Int 23(8):2093-2097

3. National Osteoporosis Foundation. Clinician's guide to prevention and treatment of osteoporosis. http://nof.org/hcp/resources/913. Accessed 18 Oct 2013

4. US Department of Health and Human Services. Bone health and osteoporosis: a report of the surgeon general. http://www.surgeongeneral. gov/library/reports/bonehealth/. Accessed 18 Oct 2013
5. Watts NB, Bilezikian JP, Camacho PM et al (2010) American Association of Clinical Endocrinologists medical guidelines for clinical practice for the diagnosis and treatment of postmenopausal osteoporosis. Endocr Pract 16(Suppl 3):1-37

6. Sweet MG, Sweet JM, Jeremiah MP, Galazka SS (2009) Diagnosis and treatment of osteoporosis. Am Fam Physician 79(3):193-200

7. Lewiecki EM, Laster AJ, Miller PD, Bilezikian JP (2012) More bone density testing is needed, not less. J Bone Miner Res 27(4):739-742

8. Wainwright SA, Marshall LM, Ensrud KE et al (2005) Hip fracture in women without osteoporosis. J Clin Endocrinol Metab 90(5):27872793

9. Lindsay R, Silverman SL, Cooper C et al (2001) Risk of new vertebral fracture in the year following a fracture. JAMA 285(3): 320-323

10. Gehlbach S, Saag KG, Adachi JD et al (2012) Previous fractures at multiple sites increase the risk for subsequent fractures: the global longitudinal study of osteoporosis in women. J Bone Miner Res 27(3):645-653

11. Edwards BJ, Bunta AD, Simonelli C, Bolander M, Fitzpatrick LA (2007) Prior fractures are common in patients with subsequent hip fractures. Clin Orthop Relat Res 461:226-230

12. Schuit SCE, van der Klift M, Weel AE et al (2004) Fracture incidence and association with bone mineral density in elderly men and women: the Rotterdam Study. Bone 34(1):195-202

13. Siris ES, Chen Y, Abbott TA et al (2004) Bone mineral density thresholds for pharmacological intervention to prevent fractures. Arch Intern Med 164(10):1108-1112

14. Barrett-Connor E, Sajjan SG, Siris ES, Miller PD (2008) Wrist fracture as a predictor of future fractures in younger versus older postmenopausal women: results from the National Osteoporosis Risk Assessment (NORA). Osteoporos Int 19(5):607-613

15. Kanis J (2008) Assessment of osteoporosis at the primary healthcare level. World Health Organization Collaborating Centre, University of Sheffield, Sheffield, UK

16. Tosteson AN, Melton LJ 3rd, Dawson-Hughes B et al (2008) Costeffective osteoporosis treatment thresholds: the United States perspective. Osteoporos Int 19(4):437-447

17. Black DM, Delmas PD, Eastell R et al (2007) Once-yearly zoledronic acid for the treatment of postmenopausal osteoporosis. N Engl J Med 356(18):1809-1822

18. Lyles KW, Colon-Emeric CS, Magaziner JS et al (2007) Zoledronic acid and clinical fractures and mortality after hip fracture. N Engl $\mathrm{J}$ Med 357(18):1799-1809

19. Neer RM, Arnaud CD, Zanchetta JR et al (2001) Effect of parathyroid hormone (1-34) on fractures and bone mineral density in postmenopausal women with osteoporosis. N Engl J Med 344(19):1434 1441

20. McCloskey EV, Johansson H, Oden A et al (2009) Ten-year fracture probability identifies women who will benefit from clodronate therapy-additional results from a double-blind, placebo- controlled randomised study. Osteoporos Int 20(5):811-817

21. Donaldson MG, Palermo L, Ensrud K, Hochberg MC, Schousboe JT, Cummings SR (2012) Effect of alendronate for reducing fracture by FRAX score and femoral neck bone mineral density: the Fracture Intervention Trial. J Bone Miner Res 27(8):1804-1810

22. Burge R, Dawson-Hughes B, Solomon DH, Wong JB, King A, Tosteson A (2007) Incidence and economic burden of osteoporosisrelated fractures in the United States, 2005-2025. J Bone Miner Res 22(3):465-475 